\begin{tabular}{ll|l}
\cline { 2 - 3 } & \multicolumn{2}{l}{ Intervent Neurol 2016;5:76-80 } \\
\cline { 2 - 3 } & $\begin{array}{l}\text { DOI: 10.1159/000444507 } 2016 \text { S. Karger AG, Basel } \\
\text { Published online: May 12, 2016 }\end{array}$ & $\begin{array}{l}\text { (C) } 1664-9737 / 16 / 0052-0076 \$ 39.50 / 0 \\
\text { www.karger.com/ine }\end{array}$ \\
\hline
\end{tabular}

\title{
Endovascular Management of Sigmoid Sinus Diverticulum
}

\author{
Srinivasan Paramasivam ${ }^{a} \quad$ Sunil Furtado ${ }^{a} \quad$ Tomoyoshi Shigamatsu $^{a}$ \\ Eric Smouhab \\ ${ }^{a}$ Center for Cerebrovascular Surgery, Department of Neurosurgery, and ${ }^{b}$ Department of \\ Otolaryngology, Mount Sinai Hospital, New York, N.Y., USA
}

\section{Key Words}

Pulsatile tinnitus · Sigmoid sinus diverticulum · Sinus stenosis

\begin{abstract}
Sigmoid sinus diverticulum (SSD) is a rare vascular disorder due to dehiscence of the sigmoid plate. It may be associated with prediverticular venous sinus stenosis (SS) and usually presents as pulsatile tinnitus. The mechanism of development of the SSD and tinnitus from a sinus diverticulum and associated SS is unclear. Previous case reports have suggested that remodeling of the venous system targeting the stenosis, elimination of the diverticulum, or both, have resulted in symptom relief. We present a case of SSD with SS, treated by stenting of the stenosis along with coil embolization of the diverticulum, resulting in complete relief of symptoms. We have also reviewed the literature and discussed the evolution of management from open surgical treatment to endovascular treatment.

(C) 2016 S. Karger AG, Basel
\end{abstract}

\section{Introduction}

Pulsatile tinnitus (PT) is a symptom of varied etiology, mostly caused by vascular pathology in and around the ear. In most cases, the etiology is identified using CT, a CT angiogram, MRI and digital subtraction angiography (DSA). Sigmoid sinus diverticulum (SSD) is identified as one of the rare causes of PT [1]. The management of SSD involves reconstruction of the sigmoid sinus wall and has changed over the years from open surgical treatment to endovascular treatment. 


\begin{tabular}{l|l}
\hline DOI: $10.1159 / 000444507$ & $\begin{array}{l}\text { ○ } 2016 \text { S. Karger AG, Basel } \\
\text { www.karger.com/ine }\end{array}$ \\
\hline
\end{tabular}

Paramasivam et al.: Endovascular Management of Sigmoid Sinus Diverticulum

Fig. 1. a, b CT scan (a) and bone window (b) reveal the dehiscence of the bony wall leading to the SSD (arrows). c, d MR venograms in anteroposterior and lateral projection reveal stenosis of the right transverse sigmoid sinus with the SSD (arrows). There is also hypoplasia of the left transverse sigmoid sinus.
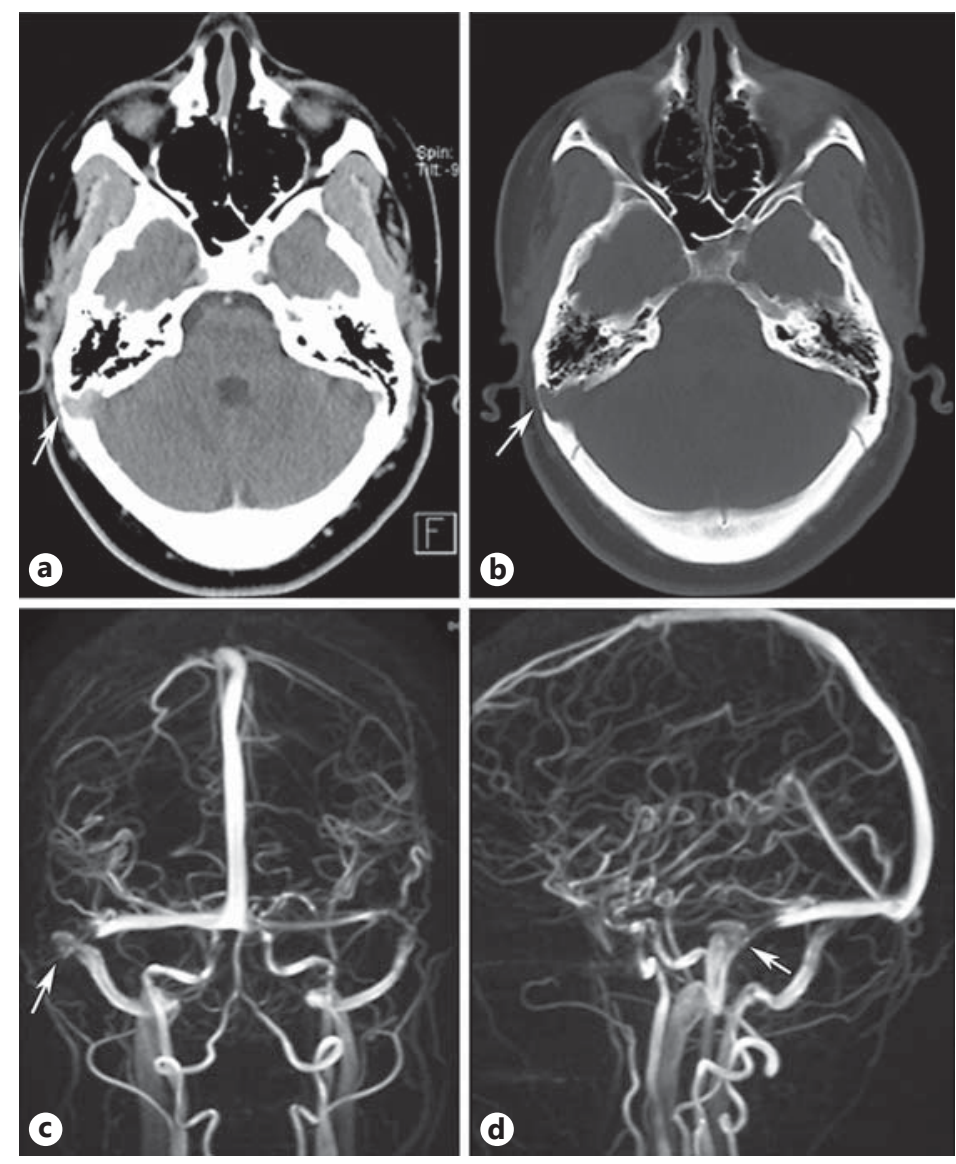

\section{Case Report}

Our patient is an obese female in her late thirties, who presented with persistent disturbing noise in her right ear of 4 months duration. The noise became more obvious in a quiet environment and with physical exertion. She had no change in hearing, balance and coordination. She had normal neurologic, otologic and neuro-ophthalmologic evaluations. Subsequent noninvasive imaging included carotid ultrasound, CT of the brain with bone window, and MRI with MR venography of the brain that revealed dehiscence of the sigmoid plate with a diverticulum of the venous sinus protruding into the mastoid air cells on the right side with a hypoplastic left transverse sigmoid sinus. An upstream stenosis at the transverse sigmoid junction just proximal to the stenosis was noted (fig. 1). DSA identified the right SSD measuring about $7 \times 5 \mathrm{~mm}$, showing a broad base with prediverticular stenosis at the transverse sigmoid sinus. Hypoplasia of the left transverse sigmoid sinus was also noted (fig. 2).

The patient was premedicated with aspirin and Plavix, and platelet function was tested before the procedure. Under general anesthesia, transvenous access was obtained and a Neuron Max catheter was advanced into the right internal jugular vein. A Protégé stent system of $10 \times 30 \mathrm{~mm}$ followed by a Protégé stent of $10 \times 40 \mathrm{~mm}$ was deployed in overlapping fashion. The diverticulum was coiled using 3 Target 360 Ultra coils resulting in total obliteration of the diverticula. Following the procedure, the patient was completely relieved of the symptoms. At 8 months follow-up, she was symptom free, and a follow-up angiogram revealed remodeling of the right transverse sigmoid sinus and persistent obliteration of the diverticulum (fig. 3). 

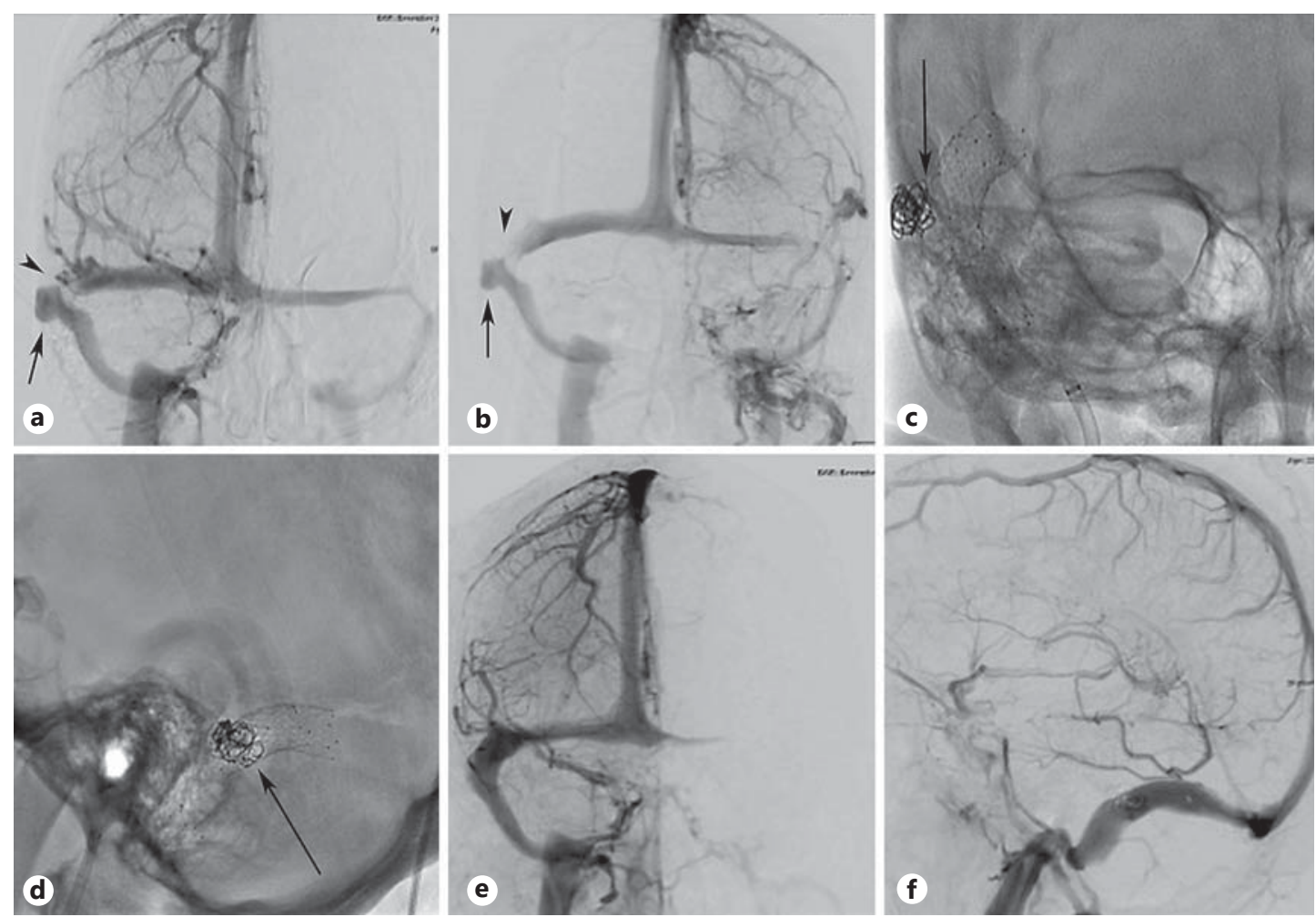

Fig. 2. a, b Angiograms of the right internal carotid (a) and the left internal carotid arteries (b) in anteroposterior view reveal the right SSD (arrows) and proximal stenosis in the distal transverse sinus (arrowheads). c, $\mathbf{d}$ Posttreatment native images reveal the stent in the transverse sigmoid sinus and coils in the diverticulum (long arrow). e, f Right internal carotid artery angiograms reveal remodeling of the sinus with obliteration of the diverticulum.

Fig. 3. Follow-up angiogram done at 8 months: native images $(\mathbf{a}, \mathbf{b})$ reveal stable stent and coils (arrows), and right internal carotid angiograms (c, d) reveal stable and complete obliteration of the diverticulum with right transverse sigmoid sinus remodeling (arrowheads).
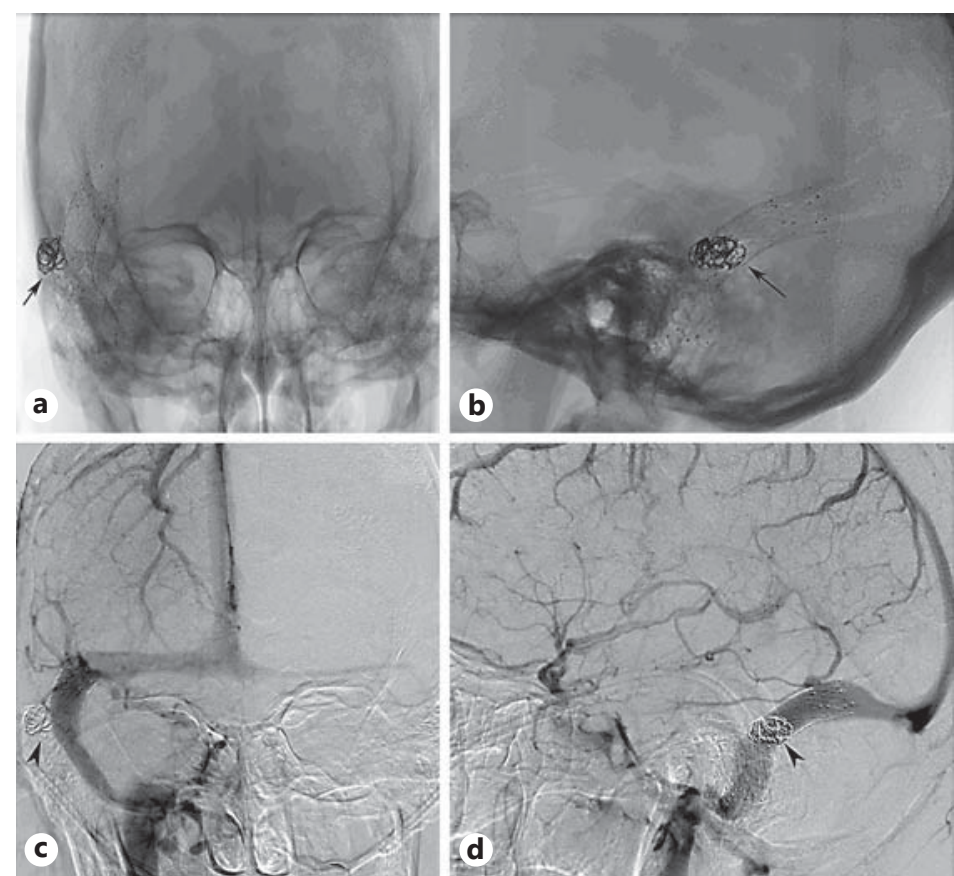
Table 1. Summary of case reports of SSD treated by endovascular embolization

\begin{tabular}{llllll}
\hline First author [Ref.], year & $\begin{array}{l}\text { Age, } \\
\text { years/sex }\end{array}$ & Side/dominancy & Treatment & $\begin{array}{l}\text { Upstream } \\
\text { stenosis }\end{array}$ & Antiplatelet medication \\
\hline Houdart [1], 2000 & $33 / \mathrm{F}$ & Left/dominant & Simple coiling & $(+)$ & ASA 160 mg \\
Sanchez [7], 2002 & $54 / \mathrm{M}$ & Left/unknown & Stent-assisted coiling & $(+)$ & Not mentioned \\
Zenteno [8], 2004 & $38 / \mathrm{F}$ & Left/dominant & Stent-assisted coiling & $(+)$ & ASA 325 mg + CLO 75 mg \\
Gard [9], 2009 & $48 / \mathrm{F}$ & Left/dominant & Simple coiling & $(-)$ & ASA 325 mg \\
Mehanna [10], 2010 & $46 / \mathrm{F}$ & Right/dominant & Balloon-assisted coiling & $(-)$ & ASA 325 mg \\
Park [11], 2011 & $31 / \mathrm{F}$ & Right/dominant & Simple coiling & $(-)$ & None \\
Santos-Franco [12], 2012 & $59 / \mathrm{F}$ & Right/dominant & Stenting alone & $(+)$ & ASA 100 mg + CLO 75 mg \\
Amans [13], 2014 & $59 / \mathrm{F}$ & Right/dominant & Simple coiling & $(+)$ & ASA 81 mg \\
Present case, 2015 & $33 / \mathrm{F}$ & Right/dominant & Stent-assisted coiling & $(-)$ & ASA 81 mg + CL0 75 mg \\
\hline \multicolumn{2}{c}{ ASA = Asprin; CLO clopidogrel. } & & & & \\
\hline
\end{tabular}

\section{Discussion}

A tinnitus may be subjective or objective and may be pulsatile or nonpulsatile. In most cases, PT is caused by vascular pathology that may have arterial or venous causes, and it is generated by turbulent flow of blood through stenotic or irregular vascular structures in and around the petrous bone. Like any solid structure, the petrous bone is a good conductor of sound and transmits to the middle ear. The sound becomes more rapid and is often louder with physical exertion. Evaluation for causes of PT includes a high-resolution cranial CT scan, CT angiography, MRI, MR angiography and MR venography examination of the head, carotid Doppler scans and DSA. Sonmez et al. [2] have detected the etiology of PT using imaging in $67.6 \%$ of cases and $100 \%$ of the time when the tinnitus was objective.

SSD is a rare venous anomaly associated with PT; however, the exact incidence and mechanisms of generation are unknown. In about half the cases, the SSD is present on the side of the dominant transverse sigmoid sinus, being at least $3 \mathrm{~mm}$ larger than the other [3]. This condition is reported more commonly in middle-aged women [3]. It is due to dehiscence of the wall of the sinus and protrusion of part of the sinus into the pneumatized bone, resulting in pulsatile and potentially disabling tinnitus [4]. Upstream sinus stenosis (SS) is often noticed with SSD and has been considered to have a causative role in sinus diverticulum generation, as in our case. Compression of the ipsilateral internal jugular vein eliminates or attenuates venous PT, with its loudness often influenced by head position [3]. Dural SS per se can cause PT due to turbulent flow of blood through stenotic vascular structures or secondary to intracranial hypertension caused by venous hypertension [4, 5]. Since not all SSD causes PT, in symptomatic patients, treatment of either the stenosis or the diverticulum, or both, remains controversial.

Successful surgical treatment has been described; it involves extended mastoidectomy, skeletonization of the affected sinus wall, reduction of the diverticulum with bipolar cautery, reinforcement of the sinus wall using soft tissue graft of temporalis fascia and reconstruction of the bony defect [5]. We acquired the knowledge of dural sinus stenting from benign intracranial hypertension [6].

Endovascular treatment of SSD and SS is feasible with coil embolization with or without stent assistance. We found seven case reports describing endovascular management of SSD as a treatment of PT (table 1) [1,7-13]. Three of these cases received a stent with or without coiling $[7,8,12]$. All patients needing sinus stenting will have to be premedicated with dual 
antiplatelet medication. In order to minimize procedural risks, risk of in-stent thrombosis and hemorrhagic complications, stents should be limited to cases requiring their use to adequately position coils in the diverticulum, as in our case and a previous one [8].

When the SSD with upstream SS is identified, treatment of the stenotic lesion either by balloon angioplasty alone or stenting remains unknown. Three cases of SSD with upstream SS were reported with three different endovascular treatments with symptom relief. One was treated by stenting to resolve the SS without coiling the SSD [12], another by coiling the SSD without treatment of stenois [13], and the third was treated by stenting the stenosis and coiling of the SSD [7]. In general, for patients with SS causing idiopathic intracranial hypertension, a pressure gradient of more than $10 \mathrm{~mm} \mathrm{Hg}$ is used as an indicator for sinus stenting. The pressure gradient across the stenotic segment may help in assessing the severity of stenosis, determining the need for angioplasty and stenting the upstream SS associated with SSD.

\section{Conclusion}

SSD with or without SS is a rare cause of PT. Symptomatic SSD can be safely treated by endovascular coil embolization of the diverticula with or without stenting. Management of the associated stenosis may be performed during the same procedure.

\section{Disclosure Statement}

S.P. is a consultant to Microvention. There are no disclosures for the other authors.

\section{References}

1 Houdart E, Chapot R, Merland JJ: Aneurysm of a dural sigmoid sinus: a novel vascular cause of pulsatile tinnitus. Ann Neurol 2000;48:669-671.

2 Sonmez G, Basekim CC, Ozturk E, Gungor A, Kizilkaya E: Imaging of pulsatile tinnitus: a review of 74 patients. Clin Imaging 2007;31:102-108.

3 Wang GP, Zeng R, Liu ZH, Liang XH, Xian JF, Wang ZC, et al: Clinical characteristics of pulsatile tinnitus caused by sigmoid sinus diverticulum and wall dehiscence: a study of 54 patients. Acta Otolaryngol 2014;134:7-13.

4 Krishnan A, Mattox DE, Fountain AJ, Hudgins PA: CT arteriography and venography in pulsatile tinnitus: preliminary results. AJNR Am J Neuroradiol 2006;27:1635-1638.

5 Eisenman DJ: Sinus wall reconstruction for sigmoid sinus diverticulum and dehiscence: a standardized surgical procedure for a range of radiographic findings. Otol Neurotol 2011;32:1116-1119.

6 Albuquerque FC, Dashti SR, Hu YC, Newman CB, Teleb M, McDougall CG, et al: Intracranial venous sinus stenting for benign intracranial hypertension: clinical indications, technique, and preliminary results. World Neurosurg 2011;75:648-652; discussion 592-595.

7 Sanchez TG, Murao M, de Medeiros IR, Kii M, Bento RF, Caldas JG, et al: A new therapeutic procedure for treatment of objective venous pulsatile tinnitus. Int Tinnitus J 2002;8:54-57.

8 Zenteno M, Murillo-Bonilla L, Martinez S, Arauz A, Pane C, Lee A, et al: Endovascular treatment of a transversesigmoid sinus aneurysm presenting as pulsatile tinnitus. Case report. J Neurosurg 2004;100:120-122.

9 Gard AP, Klopper HB, Thorell WE: Successful endovascular treatment of pulsatile tinnitus caused by a sigmoid sinus aneurysm. A case report and review of the literature. Interv Neuroradiol 2009;15:425-428.

10 Mehanna R, Shaltoni H, Morsi H, Mawad M: Endovascular treatment of sigmoid sinus aneurysm presenting as devastating pulsatile tinnitus. A case report and review of literature. Interv Neuroradiol 2010;16:451-454.

11 Park YH, Kwon HJ: Awake embolization of sigmoid sinus diverticulum causing pulsatile tinnitus: simultaneous confirmative diagnosis and treatment. Interv Neuroradiol 2011;17:376-379.

12 Santos-Franco JA, Lee A, Nava-Salgado G, Zenteno M, Vega-Montesinos S, Pane-Pianese C: Hybrid carotid stent for the management of a venous aneurysm of the sigmoid sinus treated by sole stenting. Vasc Endovascular Surg 2012;46:342-346.

13 Amans MR, Stout C, Dowd CF: Resolution of pulsatile tinnitus after coil embolization of sigmoid sinus diverticulum. J Cerebrovasc Dis Stroke 2014;1:1010. 